\title{
Comparative Study between Cervical Laminectomy and Cervical Laminectomy with Fixation on Clinical Outcome and Spine Stability in Patients with Degenerative Cervical Myelopathy
}

\author{
Adel Nabeeh Mohamed Abdalla, Mohamed Wael Sameer, Amr Mohamed Nageeb El-Shehaby, \\ Mohamed Abdalla El-Werdany, Mohammed Abdelaty Mohamed Abdalla
}

Department of Neurosurgery, Faculty of Medicine, Ain Shams University, Cairo, Egypt

\begin{abstract}
Background: Degenerative Cervical Myelopathy (DCM) is an age-related disease of the cervical spine and represents one of the most common causes of spinal cord dysfunction. Surgical intervention is the cornerstone of management in symptomatic cases, but the approach of choice is always debatable.

Purpose: Was to compare between muttilevel cervical laminectomy, and multilevel cervical laminectomy with lateral mass fixation in patients with cervical spondylotic myelopathy regarding the functional clinical outcome and cervical spine normal sagittal alignment.

Patients and Methods: This work is a prospective study of two groups of randomly selected patients. The first group $(n=20)$ underwent cervical laminectomy while the second group $(n=18)$ underwent cervical laminectomy with lateral mass fixation. Patients were followed up for 12 months duration using functional modified Japanese orthopedic association (mJAO)score, andmeasurement of $\mathrm{C} 2-\mathrm{C} 7$ cobb`s angle to evaluate post-operative cervical sagittal alignment.

Results: Results revealed that both approaches have better post-operative functional outcome with no significant changes on the normal cervical lordosis. Results alsoshowed no statistically significant difference in clinical outcome between the 2 groups after one year follow up.

Conclusion: Both simple multilevel posterior cervical laminectomy and posterior cervical laminectomy with lateral mass fixation are associated with improvement in the functional outcome in CSM patients with no changes in normal cervical lordotic sagittal alignment in at least 12 months follow up duration.
\end{abstract}

Key words: Degenerative cervical myelopathy, Laminectomy, Lateral mass fixation, Modified Japanese orthopedic score, Cobb`s angle.

\section{INTRODUCTION}

Cervical spondylotic myelopathy (CSM) is a progressive degenerative disease and is the most common cause of cervical spinal cord dysfunction. CSM can be due to direct compression of the spinal cord, or surrounding blood vessels, resulting in varied clinical symptoms. Spondylosis has been shown as the most common etiology for cervical myelopathy in people aged 55 years or older ${ }^{(1)}$. The surgical procedures include anterior and posterior approaches, the choice of which depend on the cervical alignment and the levels and sources of compression. In patients exhibiting preserved cervical lordosis and >3-level canal stenosis laminoplasty or laminectomy with or without fixation was performed. In the case of significant compression on the posterior side, posterior approach was also selected, even if the patients exhibited <3-level compression ${ }^{(2)}$.However, although the effectiveness of cervical laminectomy was documented repeatedly, there were still concerns over postoperative kyphotic deformity, cervical instability, and late deterioration ${ }^{(3)}$.

The aim of the current work was to compare between mutltilevel cervical laminectomy, and multilevel cervical laminectomy with lateral mass fixation in patients with cervical spondylotic myelopathy regarding the functional clinical outcome and cervical spine normal sagittal alignment.

\section{PATIENTS AND METHODS}

This prospective study included a total of 40 patients with multiple level cervical spondylotic myelopathy attending at Ain Shams University Hospitals. Approval of the ethical committee and a written informed consent from all the subjects were obtained. This study was conducted between January 2014 till January 2017.

Patients were randomly assigned into two groups (20 patients each), the first group underwent cervical laminectomy and the second group underwent cervical laminectomy with lateral mass fixation.

Inclusion Criteria: Age: 18 Years and over. Genders: Both. Symptomatic cervical myelopathy At least three level Cervical canal stenosis

Exclusion Criteria: Patients with preoperative cervical kyphosis or cervical sublaxation. Previous cervical intervention due to any cause. 
Pre-operatively all patients were evaluated by: History taking andclinical examinationsthat includedcomplete general and neurological examination. Each patient will be initially assessed clinically by modified Japanese orthopedic association (mJAO) score (table 1). Radiological Assessment:Plain X-rays or CT scans, and Magnetic Resonance Imaging (MRI) were done for all cases using T1, T2 images. Axial cuts as well as sagittal planes. Assessment of cervical spine sagittal alignment using (C2-C7) Cobb`s angle method, Cobb angles were measured using a measurement analysis software (ImageJ 1.46rJava 1.6.0_20 (32-bit)http://imagej.nih.gov/ij).

Table (1): $\quad$ Modified Japanese orthopedic association (mJAO) score ${ }^{(4)}$.

\begin{tabular}{|c|c|}
\hline Score & Description \\
\hline (A) Upper extremity (UE) motor function failure \\
\hline 0 & Cannot move hands \\
\hline 1 & Can move hands but cannot eat with a spoon \\
\hline 2 & Can eat with a spoon but cannot button up \\
\hline 3 & Can button up with great difficulty \\
\hline 4 & Can button up with slight difficulty \\
\hline 5 & Normal, no function loss \\
\hline (B) Lower extremity (LE) motor function failure \\
\hline 0 & Complete motor and sensation function los \\
\hline 1 & Sensation existing but cannot move legs \\
\hline 2 & Can move legs but cannot walk \\
\hline 3 & Can walk on a smooth surface using a support (cane \\
\hline 4 & Cor walker) \\
\hline 5 & Can use stairs using a support \\
\hline 6 & can use stairs without support \\
\hline 7 & Low intensity instability in walking but can walk \\
without support
\end{tabular}

Surgical Procedure

1) Group A (underwent cervical laminectomy only): A linear midline skin incision enough to expose from $\mathrm{C} 2$ to $\mathrm{C} 7$ is made.The musculature was dissected fromthe midline laterally to expose bony anatomy. The level of the exposure can usually be established correctly by intraoperative lateral radiograph to confirm the level. Decompressive laminectomy was done by drilling two gutters on both sides of the lamina which was then removed en-bloc after cutting the ligamentum flavum.the wound was closed in layers in standardfashion.

2) Group B (underwent cervical laminectomy with lateral mass fixation): The incision and Exposure of the bony anatomy are as Group A, but the muscles must be dissected off the bone far laterally to expose the entire lateral mass of the vertebrae to be fixed with lateral mass screws.An awel was used to make a shallow holes $1 \mathrm{~mm}$ medially and above center oflateral mass then the drill was used to drill a hole $1 \mathrm{~cm}$ deep in the lateral masses bilaterally, then insertion of 14-mmlong screws, $3.5 \mathrm{~mm}$ in diameter using the technique described by Magerl with 20 degree to 25 degree lateral and cranial angulations respectively, parallel to the joint line, to avoid injury to the vertebral artery and spinal nerve root. The facet joints at the levels to be fused were curetted and packed with bone grafts taken from the spinous processes. Fixation of the levels to be fused by two rods bilaterally with application of screws caps. Bone removal and wound closure are the same as Group A.

\section{Postoperative Follow-up:}

1. Clinical follow up: Postoperatively, the patient was examined immediately after operation and patient's myelopathy grade and functional status was evaluated after six months. The final clinical outcome was determined after at least 12 months using modified Japanese orthopedic association (mJAO) score.

2. Radiological Follow-up: Following up the patients using plain X-rays or CT scans at discharge, 6 months and at least 1 year postoperative and assessment ofcervical spine sagittal alignment using (C2-C7) Cobb`s angle method.

\section{Statistical methods:}

The collected data were coded, tabulated, and statistically analyzed using IBM SPSS statistics (Statistical Package for Social Sciences) software version 22.0, IBM Corp., Chicago, USA, 2013.Descriptive statistics were done for quantitative data as minimum and maximum of the range as well as mean $\pm \mathrm{SD}$ (standard deviation) for quantitative normally distributed data, while it was done for qualitative data as number and percentage.Inferential analyses were done for quantitative variables using Shapiro-Wilk test for normality testing, independent t-test in cases of two independent groups with normally distributed data 
and paired t-test in cases of two dependent groups with normally distributed data. In qualitative data, inferential analyses for independent variables were done using Chi square test for differences between proportions and Fisher's Exact test for variables.. The level of significance was taken at $\mathrm{P}$ value < 0.050 is significant, otherwise is non-significant.

\section{RESULTS}

The investigated groups described as follows:

Group-A: 20 cases with cervical laminectomy without fixation.

Group-B: 20 cases with cervical laminectomy with fixation but two patients were lost during follow up with total 18 analyzed patients.

The two groups were compared regarding age, sex distribution and duration of symptoms and the difference between both groups is found to be statistically insignificant as shown in table (2), No significant difference between the studied groups regarding presenting complaints as shown in figure (1) or in clinical and radiological findings among the studied groups as shown in figure (2).

Table (2): Demographic characteristics among the studied groups.

\begin{tabular}{|l|l|c|c|c|}
\hline \multicolumn{2}{|c|}{ Variables } & $\begin{array}{c}\text { Group-A } \\
(\text { N=20) }\end{array}$ & $\begin{array}{c}\text { Group-B } \\
(\mathbf{N}=18)\end{array}$ & P \\
\hline \multirow{2}{*}{$\begin{array}{l}\text { Age } \\
\text { (years) }\end{array}$} & Mean \pm SD & $54.4 \pm 7.9$ & $53.3 \pm 8.4$ & \multirow{2}{*}{0.673} \\
\cline { 2 - 4 } & Range & $43.0-71.0$ & $37.0-69.0$ & \\
\hline \multirow{2}{*}{$\begin{array}{l}\text { Sex } \\
\text { (n, \%) }\end{array}$} & Male & $13(65.0 \%)$ & $12(66.7 \%)$ & \multirow{2}{*}{$\# 0.914$} \\
\cline { 2 - 4 } & Female & $7(35.0 \%)$ & $6(33.3 \%)$ & \\
\hline $\begin{array}{l}\text { Duration of } \\
\text { symptoms } \\
\text { (months) }\end{array}$ & Mean \pm SD & $15.7 \pm 7.9$ & $17.7 \pm 8.2$ & \multirow{2}{*}{0.445} \\
\cline { 2 - 4 } & Range & $7.0-36.0$ & $6.0-36.0$ & \\
\hline
\end{tabular}

^Independent t-test, \#Chi square test

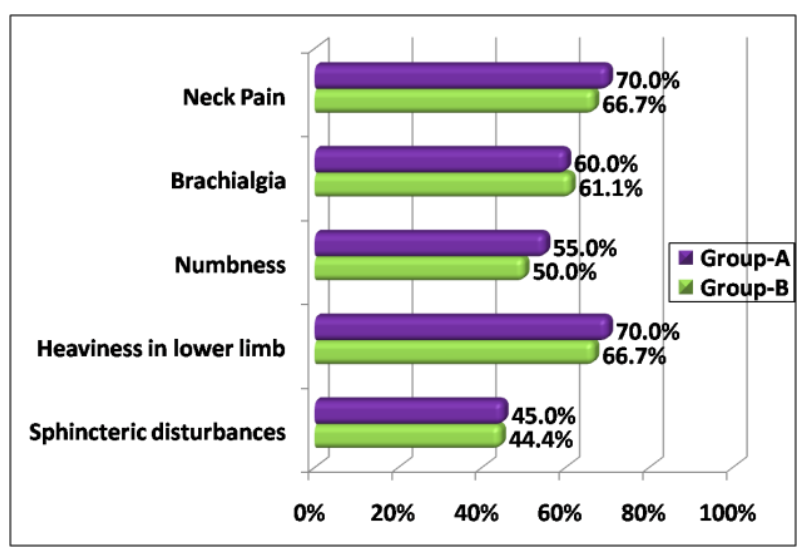

Figure (1): Presenting complaints among the studied groups.

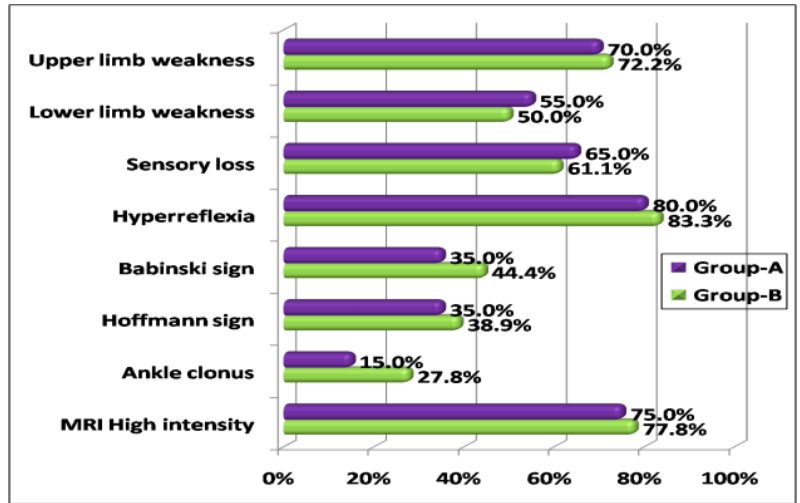

Figure (2): Basal clinical and radiological findings among the studied groups.

No significant difference between the studied groups regarding preoperative, postoperative (after 12 months follow up) and change in functional (mJAO) score. Functional (mJAO) score significantly increased in both groups after 12 months period of follow up as shown in figure (3).

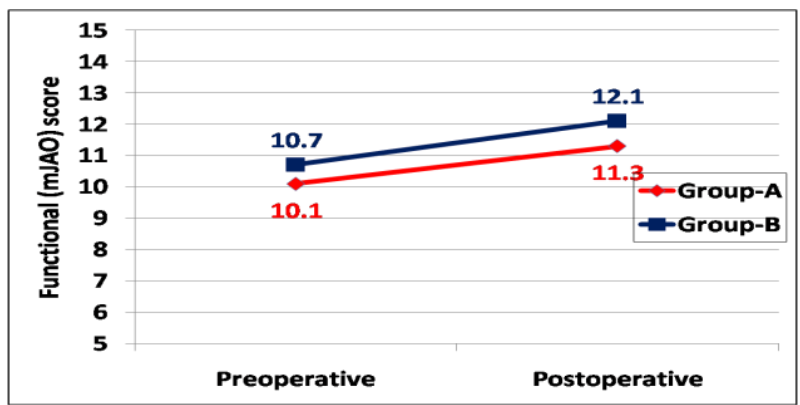

Figure (3): Functional (mJAO) score among the studied groups before and after intervention.

No significant difference between the studied groups regarding preoperative, postoperative (after 12 months follow up) and change in radiological (cobb's) angle. Radiological (cobb's) angle non-significantly decreased in both groups after 12 months period of follow up as shown in figure (4).

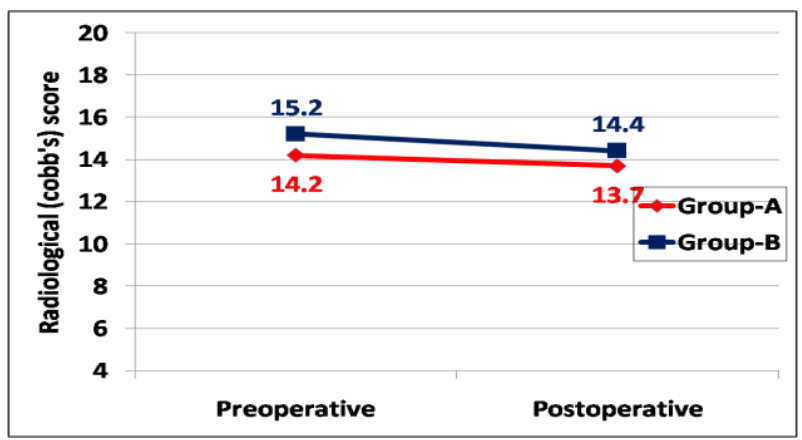

Figure (4): Radiological (cobb's) angle among the studied groups before and after intervention. 


\section{DISCUSSION}

Multilevel cervical myelopathy can be treated with either anterior, posterior, or combined anterior and posterior decompressive procedures; the choice of surgery depends on the exact location of compressive pathology, pre-operative alignment of the cervical spine, and also surgeon's preference ${ }^{(5)}$.

Simple posterior cervical decompression presented in multilevel laminectomy was initially regarded as the gold standard treatment of multilevel degenerative cervical myelopathy. But many concerns were raised regarding the effect of this procedure on spinal stability and cervical normal lordotic sagittal alignment. Laminectomy was eventually augmented to include posterior fusion. However, fusion of the cervical spine results in alteration of normal cervical biomechanics, as axial and rotational forces are no longer physiologically distributed to subjacent spinal structures which have been associated with increased rates of adjacent segment degeneration ${ }^{(6)}$.

In this study, two different posterior cervical approaches: multilevel posterior cervical laminectomy and multilevel posterior cervical laminectomy with instrumented fusion with lateral mass screws, were compared and evaluated regarding their functional outcome and their effect on the normally lordotic cervical sagittal alignment.

In our study fifty seven cases were assessed for eligibility to enroll forty cases. Seventeen cases were excluded either due to presence of preoperative kyphosis, patients had undergone previous anterior cervical spine surgery or patients refused to be enrolled in the study. The enrolled 40 cases were randomized and allocated in two groups: Group-A underwent multilevel posterior cervical laminectomy, while group-B underwent multilevel posterior cervical laminectomy with lateral mass fixation. None of group-A were lost during follow up, while two cases were lost in group-B.

In a retrospective study done in Chubu Rosai Hospital in Japan by Machino and his co-workers ${ }^{(7)}$, the prevalence of symptoms before and after surgery in a large series of patients with cervical spondylotic myelopathy (CSM) has been investigated. It was found that the preoperative prevalence of sensory function impairment in the upper and lower extremities was $88.6 \%$ and $56.5 \%$ respectively, whereas that of motor function impairment in the upper and lower extremities was $77.7 \%$ and $80.4 \%$, respectively. The preoperative prevalence of urinary bladder function impairment was $41.2 \%$. The prevalence and distribution of pre-operative symptoms in this study is similar to our study.

There was no pathognomic sign in degenerative cervical myelopathy patients in our study as similarly stated by Acharya and his colleagues $^{(8)}$, where they evaluated the prevalence of signs of myelopathy like Hoffman sign, Babinski sign, clonus, and hyperreflexia. When considered individually, Babinski extensor response $(95 \%)$ was most sensitive followed by Hoffman sign (86\%) while clonus (48\%) was poorly sensitive for detecting myelopathy.

MRI signal changes in the spinal cord of DCM patients are usually associated with the degree of neurological severity, prevalence of specific clinical manifestations, and potential for neurological recovery. In our study we noticed the presence of T2WI hyperintense signal changes in the two groups; in group-A, it was 15(75.0\%), while in group-B it was 14 (77.8\%). T2WI signal hyperintensity was seen in about three quarters of patients $(76.5 \%, 341 / 446)$ in a large study done by Nouri et al. ${ }^{(9)}$. using MRI analysis to study the prevalence and spectrum of pathologies in patients with degenerative cervical myelopathy.

The results of the study were comparable with those of similar studies done in the past. For Instance, in a study done by Lee et al. ${ }^{(10)}$, fiftyseven patients suffering from degenerative cervical spondylotic myelopathy with OPLL underwent three posterior cervical surgeries; laminoplasty, laminectomy alone, and laminectomy with fusion with lateral mass screws and rods. Clinical outcomes measured by neck disability index and visual analogue scale were evenly improved in all groups. Cervical lordosis measured by $\mathrm{C} 2-\mathrm{C} 7$ angle and cervical curvature index non-significantly decreased in all patients after follow up of 24 months duration. These findings highly match those of the present study, except for laminoplasty approach which was not included.

Multilevel posterior cervical laminectomy approach was also evaluated by a study conducted by Saoud et al. ${ }^{(11)}$. on 56 patients with cervical spondylotic myelopathy. $73.1 \%$ of patients showed functional improvement as guidedby their modified JOA functional score, two patientsshowed deterioration representing $3.8 \%$, while $23.1 \%$ of 
patients remained unchanged. They concluded that Laminectomy is a safe and effective method in treatment of CSM. With good case selection, late kyphosis and instability can be minimal. The short operation time, fewer operative complications and lower cost add to the advantages of laminectomy. This further supports our study findings with respect to laminectomy approach.

One of the limitations of this study is the relatively short-term post-operative follow up of patients (12 months). Former studies have reported the potentiality for occurrence of delayed complications following the investigated approaches after long periods of time. For example, multilevel cervical laminectomy approach may be complicated by cervical kyphosis. Meanwhile, cervical laminectomy with fusionapproach may result in adjacent segment degeneration and increase range of motion ${ }^{(12)}$. Accordingly, follow up of patients undergoing the above mentioned surgical procedures for longer duration of time is highly recommended.

\section{CONCLUSION}

It could be concluded that posterior cervical approaches are effective and safe procedures for surgical management of CSM. Both simple multilevel posterior cervical laminectomy and posterior cervical laminectomy with lateral mass fixation are associated with improvement in the functional outcome in CSM patients. No significant post-operative changes occurred in the normal cervical lordotic sagittal alignment with the two surgical approaches in at least 12 months follow up duration.

\section{REFERENCES}

1. BakhsheshianJ, Mehta V and Liu J (2017): Current Diagnosis and Managementof Cervical Spondylotic Myelopathy. Global Spine Journal, 7(6) 572-586.

2. Kong $L$, Meng $L$, Wang $L$, Shen $Y$, Wang $P$ and Shang $Z$ (2013): Evaluation of conservative treatment and timing of surgical intervention for mild forms of cervical spondylotic myelopathy. Exp Ther Med., 6(3): 852-856.

3. McAllister B,Rebholz B and Wang J (2012): Is posterior fusion necessary with laminectomy in the cervical spine? Surg Neurol Int., 3(3): S225-S231.

4. GocmezC,Koc R, Tucer B, Menku A and Kurtsoy A (2015): Prognostic Factors in Cervical Spondylotic Myelopathy: A Clinical Prospective Study. Neurosurg Q., 25:34-40.
5. Joaquim A, Ghizoni E, Tedeschi H, Hsu W and Patel A (2016): Management of degenerative cervical myelopathy - An update. Rev Assoc Med Bras., 62(9):886-894.

6. Woods B, Hohl J and Kang J (2011): Laminoplasty versus Laminectomy and Fusion for Multilevel Cervical Spondylotic Myelopathy. Clin Orthop Relat Res.,469(3): 688-695.

7. Machino M, Yukawa Y,Hida T, Ito $K$, Nakashima H,Kanbara S, Morita D and Kato F (2012): The Prevalence of Pre- and Postoperative Symptoms in Patients With Cervical Spondylotic Myelopathy treated by Cervical Laminoplasty. Spine, 37: 1383 - 1388

8. Acharya S, Srivastava A,Virmani $S$ and Tandon R (2010): Resolution of Physical Signs and Recovery inSevere Cervical Spondylotic Myelopathy AfterCervical Laminoplasty. Spine, 35:E1083-E1087.

9. Nouri, A, Martin A, Kato S, Kermani H, Riehm $L$ and Fehlings $M$ (2017): The Relationship Between MRI Signal Intensity Changes, Clinical Presentation, and Surgical Outcome in Degenerative Cervical Myelopathy: Analysis of a Global Cohort. Spine, 42:1851-1858

10. Lee C,Jahng T, Hyun S, Kim $K$ and Kim $H$ (2016): Expansive Laminoplasty Versus Laminectomy Alone Versus Laminectomy and Fusion for Cervical Ossification of the Posterior Longitudinal Ligament: Is There a Difference in the Clinical Outcome and Sagittal Alignment?. Clin Spine Surg., 29:915.

11. SaoudK, Hamad A, Saoud A and Sharaf $O$ (2006): Assessment of Instability Following Cervical Laminectomy for Cervical Spondylotic Myelopathy and its Effect on the Clinical Outcome. WSJ., 2(1):22-26.

12. Du W, Wang L, Shen Y, Zhang Y, Ding W and Ren $L$ (2013): Long-term impacts of different posterior operations on curvature, neurological recovery and axial symptoms for multilevel cervical degenerative myelopathy. Eur Spine J., 22:1594-1602. 